\title{
Lentiviral short hairpin RNA screen of human kinases and phosphatases to identify potential biomarkers in oral squamous cancer cells
}

\author{
MING-HAN YEH ${ }^{1}$, TZUNG-CHIEH TSAI ${ }^{3}$, HAN-PENG KUO ${ }^{1}$, NAI-WEN CHANG ${ }^{4}$, \\ MIAO-RONG LEE ${ }^{4}$, JING-GUNG CHUNG ${ }^{5}$, MING-HSUI TSAI ${ }^{6}$, JAH-YAO LIU ${ }^{1,7}$ and MING-CHING KAO ${ }^{1,2,5}$ \\ ${ }^{1}$ Graduate Institute of Life Sciences and ${ }^{2}$ Department of Biochemistry, National Defense Medical Center, No. 161, Section 6, \\ Min-Chuan East Road, Taipei, Taiwan 11490; ${ }^{3}$ Department of Microbiology, Immunology and Biopharmaceuticals, \\ College of Life Sciences, National Chiayi University, No. 300, Syuefu Road, Chiayi, Taiwan 60004; ${ }^{4}$ Department of \\ Biochemistry, College of Medicine and ${ }^{5}$ Department of Biological Science and Technology, College of Life Sciences, \\ China Medical University, No. 91, Hsueh-Shih Road, Taichung, Taiwan 40402; ${ }^{6}$ Department of Otolaryngology, \\ China Medical University Hospital, No. 2, Yuh-Der Road, Taichung, Taiwan 40447; ${ }^{7}$ Department of Obstetrics \\ and Gynecology, Tri-Service General Hospital, No. 325, Section 2, Chenggong Road, Taipei, Taiwan 11490, R.O.C.
}

Received April 12, 2011; Accepted May 10, 2011

DOI: 10.3892/ijo.2011.1100

\begin{abstract}
Oral carcinoma is a serious public health problem and the leading cause of head and neck cancer mortality worldwide. Moreover, oral cancer patients often present symptoms at a late stage and show a high recurrence rate after treatment. Therefore, there is an urgent need to identify novel biomarkers for early diagnosis or clinical oral cancer therapy. In this study, we employed a subset of lentiviral short hairpin RNAs targeted against various kinases and phosphatases, designed by The RNAi Consortium, to screen systemically and in a high-throughput manner for potential growth regulators of oral cancer cells. The screen revealed a total of 50 candidate genes, for which more than $90 \%$ of growth inhibition in human oral squamous cancer HSC-3 cells was obtained. Furthermore, bioinformatic analysis of these candidate genes identified transforming growth factor- $\beta$ receptor type II- and fms-related tyrosine kinase 3-related molecular pathways that are involved in NF-kB-mediated growth of HSC-3 cells. These candidate genes may be potential biomarkers for early diagnosis of oral cancer. In addition, these candidate genes represent potential targets for anticancer drug design helping to develop a personalized treatment to combat oral cancer.
\end{abstract}

Correspondence to: Dr Ming-Ching Kao, Department of Biological Science and Technology, College of Life Sciences, China Medical University, 91 Hsueh-Shih Road, Taichung, Taiwan 40402, R.O.C.

E-mail:mckao@mail.cmu.edu.tw

Dr Jah-Yao Liu, Department of Obstetrics and Gynecology, Tri-Service General Hospital, No. 325, Section 2, Chenggong Road, Taipei, Taiwan 11490, R.O.C.

E-mail: jyliu@mail.ndmctsgh.edu.tw

Key words: oral cancer, RNA interference, high-throughput screening assays, kinases, phosphoprotein phosphatases, biomarkers

\section{Introduction}

Head and neck cancer is the sixth most common type of cancer in the world. It arises from epithelial malignancies originating in paranasal sinuses, nasal cavity, oral cavity, pharynx, and larynx (1). Among these malignancies, oral squamous cell carcinoma (OSCC) is the most prevalent diagnosed malignancy and the leading cause of head and neck cancer death worldwide (2). Several risk factors for OSCC, including smoking, alcohol drinking, betel quid consumption, human papillomavirus infection, and genetic factors have been reported and well studied for many years (3-8). The early clinical diagnosis of OSCC is mainly based on screening for leukoplakia on oral cavity examination. If a precancerous lesion is present, it will subsequently be histologically classified as slight, moderate, or severe hyperplasia, or carcinoma in situ. A higher degree of dysplasia often correlates with a higher possibility that a lesion transforms into a malignant tumor (9). However, a number of studies suggest that this prediction criterion is not reliable enough; in particular, early genetic changes may not necessarily result in observable changes in morphology (10-12). Therefore, identifying novel biomarkers for early diagnosis of OSCC may open up novel therapeutic strategies and/or improve the efficacy of therapeutic treatment.

It is well known that genetic alterations are the defining features of cancer and disturb the signal transduction network in cancer cells, resulting in inappropriate cellular growth, survival and death (13). Protein kinases and phosphatases are important regulators of cell signaling pathways by governing reversible phosphorylation. Deregulation of kinase or phosphatase activities by genetic alterations causes malignant transformation. Hence, identification of novel kinases and phosphatases responsible for those aberrant cell behaviors will significantly advance our understanding of human oral oncogenesis, subsequently leading to more effective treatments and novel therapeutic strategies 
$(14,15)$. For example, epidermal growth factor receptor (EGFR) has been identified to be amplified in $31 \%$ of OSCC patients and correlates with poor clinical outcome. Targeting of EGFR with either a monoclonal antibody against EGFR or a small-molecule EGFR tyrosine kinase inhibitor has been successfully utilized for therapeutic purposes $(16,17)$.

Currently, it is believed that personalized treatment is the major cancer therapy strategy in the future. Thus, identification of biomarkers of OSCC, such as kinases and phosphatases, is required to achieve this goal (18). To identify kinases and phosphatases for early diagnosis or as therapeutic targets for OSCC, we employed 'anti-kinome' and 'anti-phosphatome' lentiviral short hairpin RNA (shRNA) subset to perform high-throughput screening for growth regulators of human OSCC cell line HSC-3. Furthermore, the possible cellular pathways for these OSCC-related regulators were also investigated.

\section{Materials and methods}

Cell culture. Human OSCC cell line HSC-3 was obtained from the Japanese Collection of Research Bioresources. HSC-3 cells were cultured in DMEM/F12 medium (Gibco) supplemented with $10 \%$ FBS (Hyclone) and maintained at $37^{\circ} \mathrm{C}$ in a humidified atmosphere with $5 \% \mathrm{CO}_{2}$.

Lentivirus-based shRNA high-throughput screening. The VSV-G pseudotyped lentivirus-based human kinase and phosphatase subset (KP subset) was obtained from the National RNAi Core Facility (NRCF) located at the Institute of Molecular Biology/ Genomic Research Center, Academia Sinica (Taipei, Taiwan), supported by the National Research Program for Genomic Medicine Grants of NSC (NSC 97-3112-B-001-016). The lentivirus-based shRNA system from NRCF was adopted from The RNAi Consortium (TRC) (19). TRC designed mutiple distinct shRNA clones to target each gene, and shRNA oligonucleotides were constructed into lentiviral vector pLKO.1-puro to produce VSV-G pseudotyped lentivirus. The KP subset covered 1236 genes in total, including 737 kinases, 209 phosphatases and 30 genes with dual function. Conversion of relative infection unit (RIU) for HSC-3 cells and lentivirus-based shRNA highthroughput screen were adopted by following the protocol of TRC. In brief, for a single shRNA clone, HSC-3 cells were seeded in DMEM/F12 medium in the day before lentivirus transfection at $3 \times 10^{3}$ cells per well in 96-well plates. After $24 \mathrm{~h}$, medium was removed and replaced with fresh DMEM/F12 medium supplemented with $10 \mu \mathrm{g} / \mathrm{ml}$ polybrene, and then lentivirus was added to cells $(\mathrm{MOI}=3)$. Medium was removed $24 \mathrm{~h}$ post-infection and cells were washed with DPBS. Subsequently, fresh HSC-3 cells growth medium supplemented with $5 \mu \mathrm{g} / \mathrm{ml}$ puromycin was added. After $48 \mathrm{~h}$, medium was removed and replaced with fresh HSC- 3 cells growth medium and Cell Counting Kit- 8 solution (CCK-8, Dojindo). The plate was incubated for $3 \mathrm{~h}$ and then the absorbance at $450 \mathrm{~nm}$ was measured. Each shRNA clone infection was performed in duplicate, in two independent 96-well plates. Absorbance values were normalized for each shRNA clone and the average of the duplicates determined to obtain the average percentage of growth inhibition relative to the control.

Bioinformatics analysis. Candidate genes were analyzed using MetaCore $^{\mathrm{TM}}$ (GeneGo) as described (20).
shRNA transfection. Liposome-mediated transfection of shRNA was performed as described (21). Briefly, cells were seeded the day before shRNA transfection. On the day of transfection, plasmids containing shRNA cassettes and Lipofectamine 2000 (Invitrogen) were separately diluted into Opti-MEM medium (Invitrogen), mixed and incubated for $20 \mathrm{~min}$ at room temperature. The resulting lipoplex complex was then added to the cells. After incubation for $6 \mathrm{~h}$, lipoplexes were removed and replaced with fresh DMEM/F12 medium. Cells were harvested for further assays at the indicated time-point.

Viability assay. Cell viability assay was performed using 96-well dish and CCK-8 reagent (Dojindo). Cells were incubated with CCK-8 solution at $37^{\circ} \mathrm{C}$ for $3 \mathrm{~h}$ after transfection with shRNA or treatment with specific tyrosine kinase inhibitor LY-364947 (Sigma-Aldrich) or Sunitinib malate (BioVision), respectively. Then the absorbance of each well was measured at $450 \mathrm{~nm}$ by ELISA reader.

Real-time PCR. Total RNA was isolated $48 \mathrm{~h}$ after transfection from HSC-3 cells by using TRIzol reagent (Invitrogen) and converted into cDNA by using the High-Capacity cDNA Reverse Transcription Kit (Applied Biosystems). Real-time PCR reactions were performed by using Power SYBR Green Master Mix (Applied Biosystems). Sequences of FLT3 primers (sense: 5'-TCAAGTGCTGTGCATACAATTCCC-3', antisense: 5'-CACCTGTACCATCTGTAGCTGGCT-3') are as previously described (22).Primers ofTGFBR2 (sense: 5'-GGGGAAACAA TACTGGCTGA-3', antisense: 5'-GAGCTCTTGAGGTCCCT GTG-3'), IKBKB (sense: 5'-GCTGCAACTGATGCTG ATGT-3', antisense: 5'-TGTCACAGGGTAGGTGTGGA-3'), SHC1 (sense: 5'-GCCGAGTATGTCGCCTATGT-3', antisense: 5'-GGGTGGGTT-CCTGAGGTATT-3'), SMAD4 (sense: 5'-CCATTTCCAATCATCCTGCT-3', antisense: 5'-ACCT TTGCCTATGTGCAACC-3') and the internal control GAPDH gene (sense: 5'-AATGGAAATCCCATCACCATCTT-3', antisense: 5'-CATCGCCCCACTTGATTTTG-3') were designed using the Primer Express ${ }^{\mathrm{TM}}$ software (Applied Biosystems) to specifically amplify the indicated genes. Amplification reactions and data analysis were performed according to the manufacturer's instructions in an ABI PRISM 7900 instrument (Applied Biosystems). The comparative CT method was used for relative quantification of gene expression.

Western blot analysis. Western blot analysis was conducted as previously described (23). Monoclonal antibodies against TGFBR2, FLT3, IKBKB, SHC1, phospho-SHC1 (Y317) and SMAD4 were purchased from Abcam, while anti-phosphoIKBKB (S177) was purchased from Cell Signaling Technology. The anti- $\beta$-actin monoclonal antibody was purchased from Chemicon. The secondary antibodies horseradish peroxidase (HRP)-linked goat anti-mouse IgG and HRP-linked goat antirabbit IgG were purchased from Santa Cruz Biotechnology and Cell Signaling Technology, respectively.

Immunofluorescence microscopy. To investigate the subcellular localization of nuclear factor- $\mathrm{\kappa B}$ (NF- $\mathrm{\kappa B}$ ), HSC-3 cells were fixed and permeabilized according to the manufacturer's instructions (Invitrogen). Briefly, HSC-3 cells were transfected with indicated shRNA for $48 \mathrm{~h}$, fixed by incubation for $20 \mathrm{~min}$ 


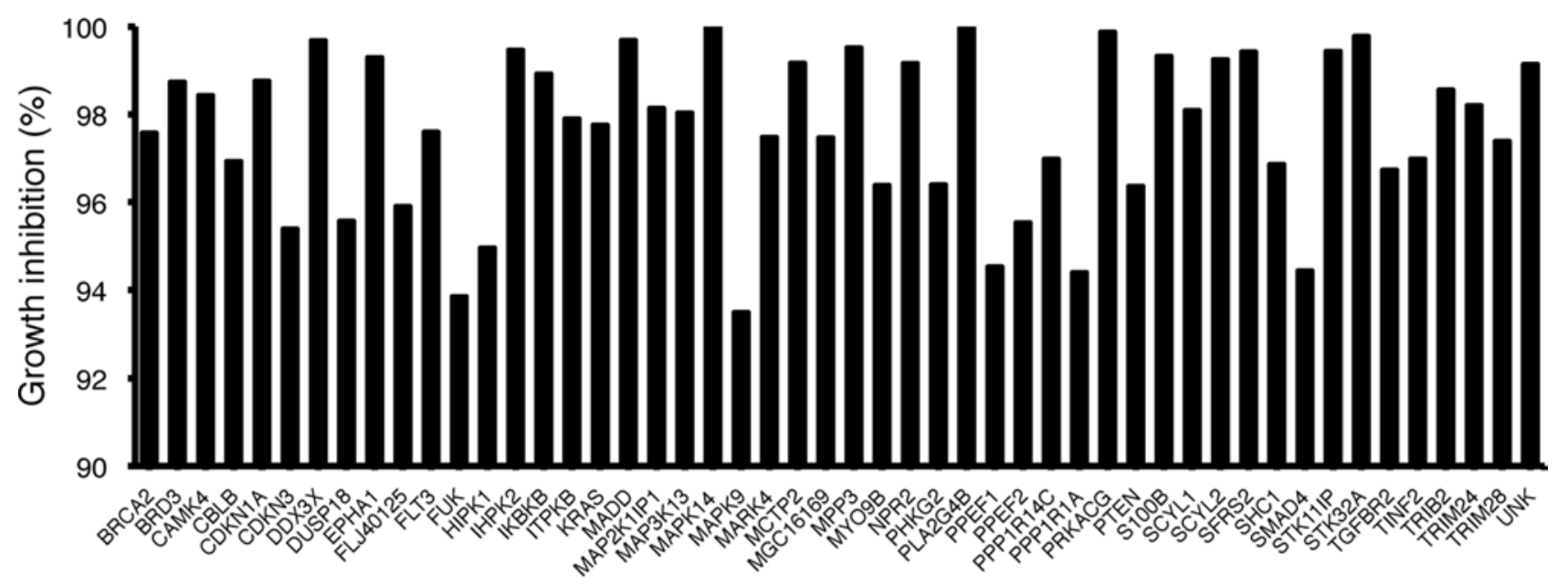

Gene

Figure 1. Putative genes with growth inhibition as identified through lentivirus-based shRNA high-throughput screening. In total, 50 genes (equivalent to 116 shRNA clones) were selected as candidates. Each gene had more than two shRNA clones with growth inhibition effect of $>90 \%$ of HSC-3 cells when compared with negative control shLuc. Each bar represents the mean of growth inhibition effect of each shRNA clone on HSC-3 cells.

at room temperature in DPBS containing $4 \%$ formaldehyde, and then permeabilized by incubation for $20 \mathrm{~min}$ on ice in DPBS containing $0.2 \%$ Triton X-100. Fixed cells were blocked with $5 \%$ bovine serum albumin (Sigma-Aldrich) and incubated with anti-NF- $\kappa$ B monoclonal antibodies (Santa Cruz) for $1 \mathrm{~h}$, followed by incubation with goat anti-rabbit Alexa fluor ${ }^{\circledR} 488$ secondary antibody (Invitrogen) for $1 \mathrm{~h}$ with washing in between. Finally, cells were stained with 4',6-Diamidino-2-phenylindole dihydrochloride (DAPI; Sigma-Aldrich) for $10 \mathrm{~min}$ at room temperature. Subcellular localization of NF- $\kappa \mathrm{B}$ was observed by using a Leica TCS SP2 confocal microscope (Leica Microsystems).

Biohazard. All experiments involving lentivirus-based shRNA were performed in a P2 Lab under the Institutional Biosafety Guideline.

Statistical analysis. The quality of screening results were determined by using the $\mathrm{Z}$ factor (24). The criteria of potential candidate genes used a $Z$ factor cutoff of $>0$. All data were analyzed by using the paired Student's t-test for comparison of independent means. $\mathrm{P}<0.05$ (two-tailed) was considered to be significant.

\section{Results}

Lentivirus-based shRNA high-throughput screen to identify genes required for growth of oral cancer cells. To identify genes involved in growth regulation of human oral cancer cells, we screened human OSCC cell line HSC-3 by using a lentivirusbased shRNA subset against human kinases and phosphatases. $\mathrm{NRCF}$ determined the relative viral titer with regard to the A549 cell line. Because different cell lines might respond differently to VSV-G pseudotyped lentivirus, we converted the RIU from A549 to HSC-3 cells. To this end, we generated a line chart of HSC-3 cell viability versus a serially diluted titer of control shLuc virus and then established a standard curve for lentivirus transfection titer. Next, we converted the RIU for
HSC-3 cells and determined the virus titer and MOI according to the standard curve. Then, based on the converted RIU, we adopted the lentivirus-based shRNA high-throughput screening and analyzed the viability of HSC-3 cells after virus infection for $72 \mathrm{~h}$. The preliminary screening results, as shown in Fig. 1, yielded 50 genes (equivalent to 116 shRNA clones), which were then selected as candidates for further evaluation ( $4 \%$ hit rate). To recognize potential off-target effects of shRNA, two or more shRNAs for each gene were used. Only genes, for which growth of HSC -3 cells was significantly inhibited $(>90 \%)$ by at least two shRNA clones with a $\mathrm{Z}$ factor $>0$, were selected as candidates. The information of these candidate shRNA clones are listed in Table I. Then, bioinformatics analysis was conducted to study the relationship between these candidate genes.

Bioinformatics analysis reveals potential biomarkers and molecular pathways that are involved in growth of oral cancer. To further determine the molecular signaling pathways regulating the growth of HSC-3 cells, all candidate genes were subjected to GeneGo MetaCore analysis. Fig. 2A shows the results from the shortest path algorithm of Dijkstra to obtain the closely related signaling molecules (25). Based on the results of MetaCore analysis, we propose that TGFBR2 and FLT3 regulate the growth of HSC-3 cells through activating SHC1 and IKBKB via phosphorylation followed by activation of their downstream signaling pathways, resulting in $\mathrm{NF}-\kappa \mathrm{B}$ translocation from cytoplasm to nucleus then transcribing genes that promote growth of HSC-3 cells. According to our results and several other reports (26-28), a putative molecular pathway is proposed in Fig. 2B.

Validation of the candidate genes. We then performed the validation for the specificity of candidate shRNAs and the correlations between shRNA-mediated gene knockdown and phenotype of cell growth inhibition. According to the results of the bioinformatics analysis, the major components (i.e., TGFBR2, FLT3, SHC1 and IKBKB) of this putative molecular signaling pathway and some relevant candidate genes (i.e., 


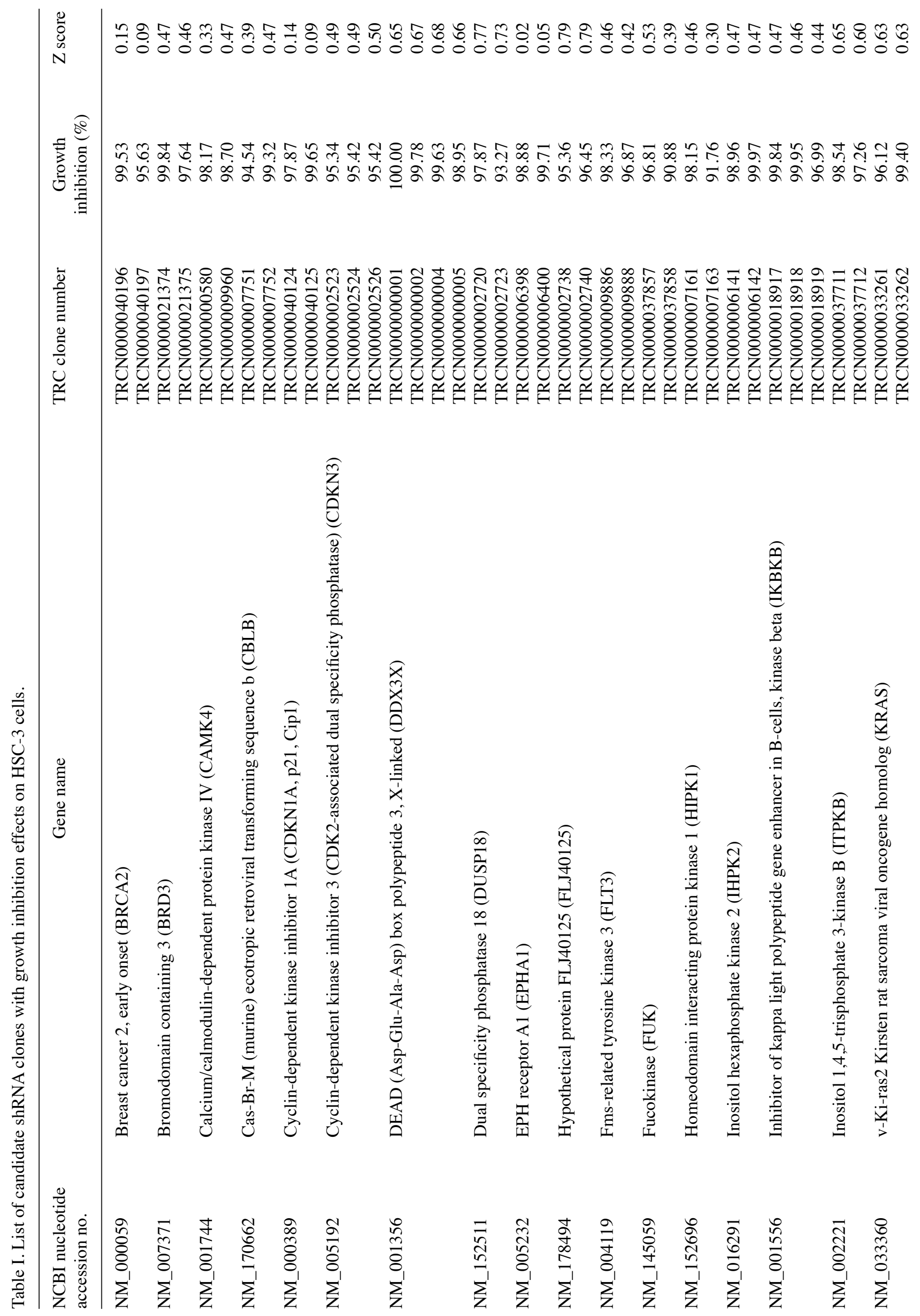




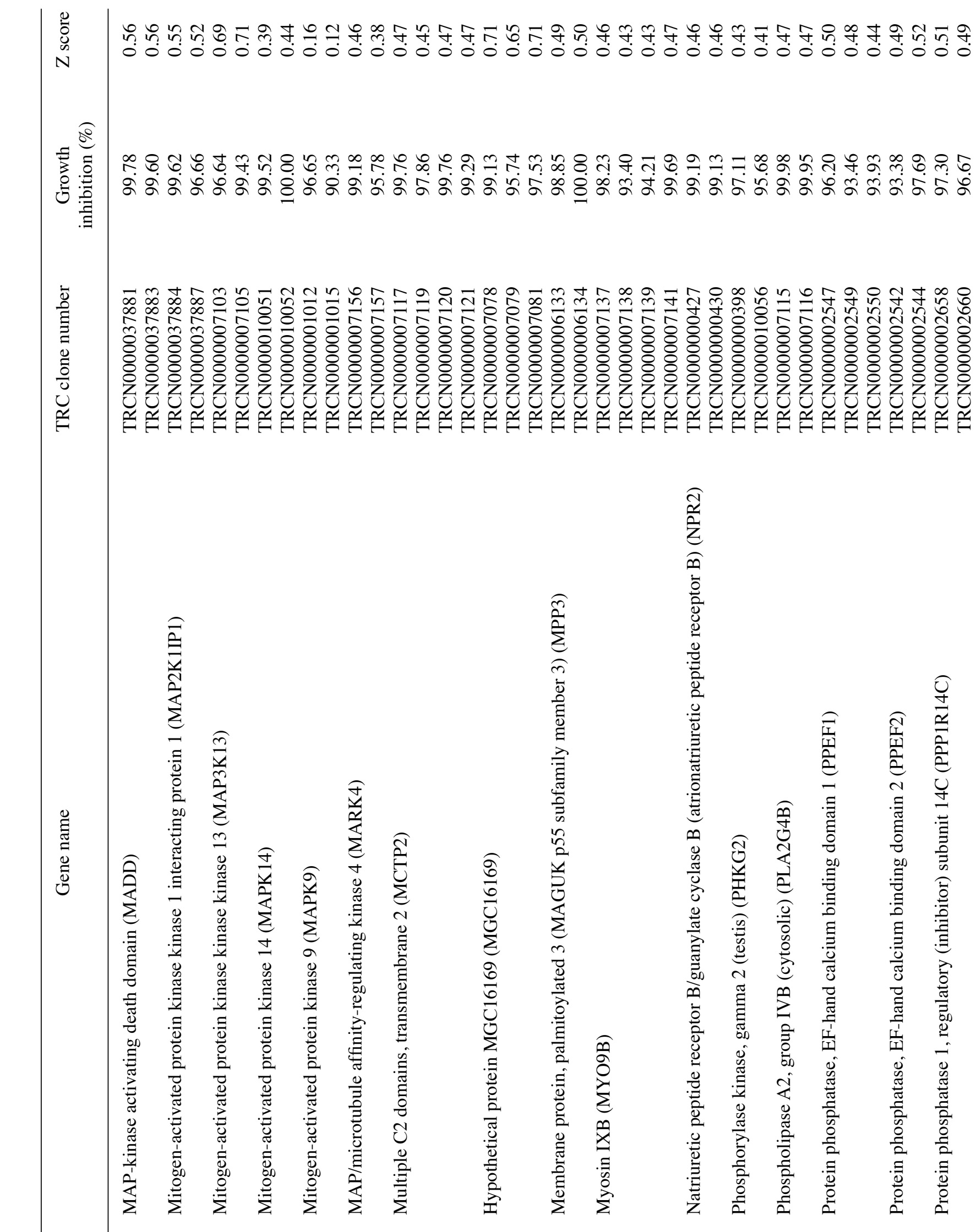




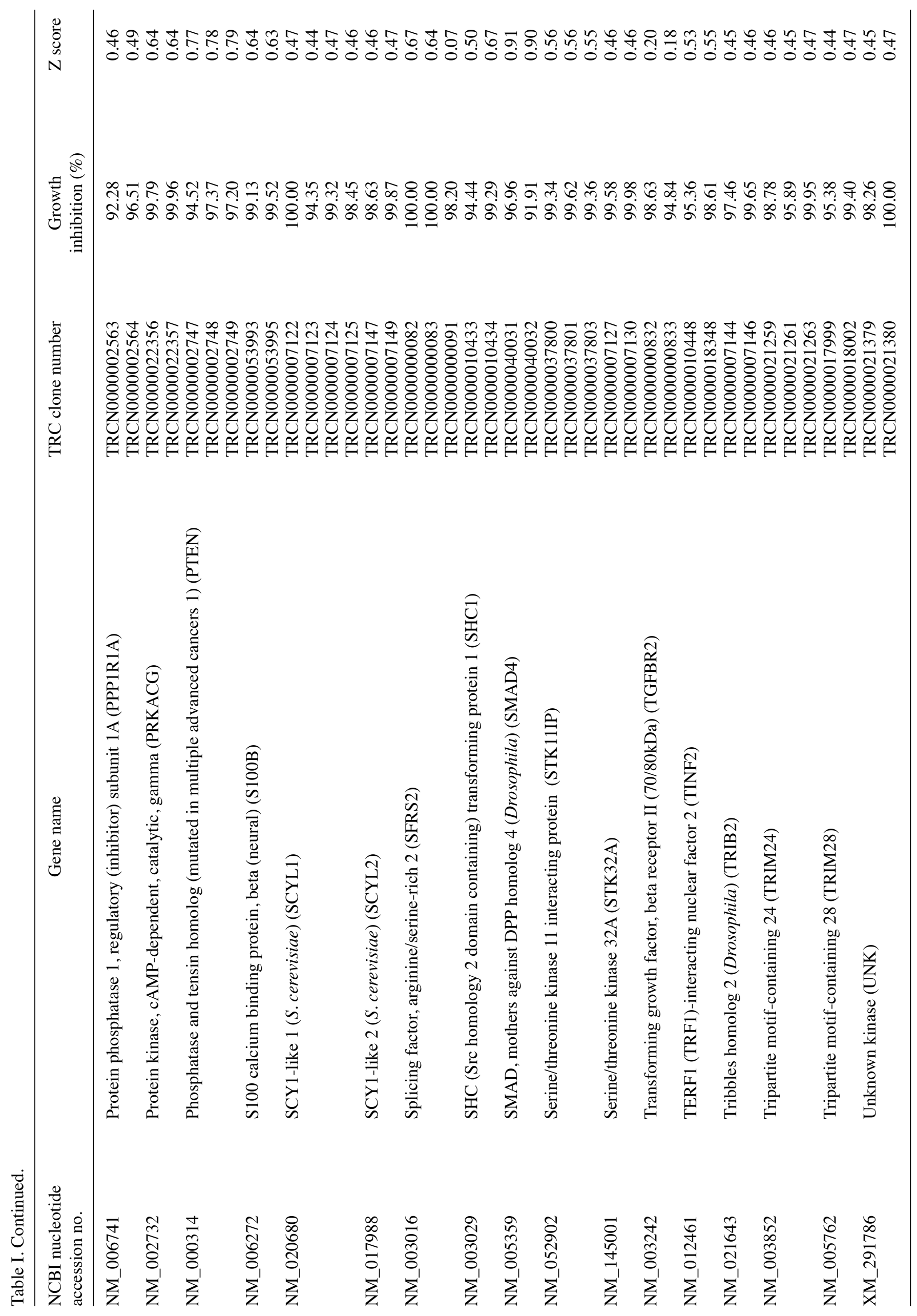


A

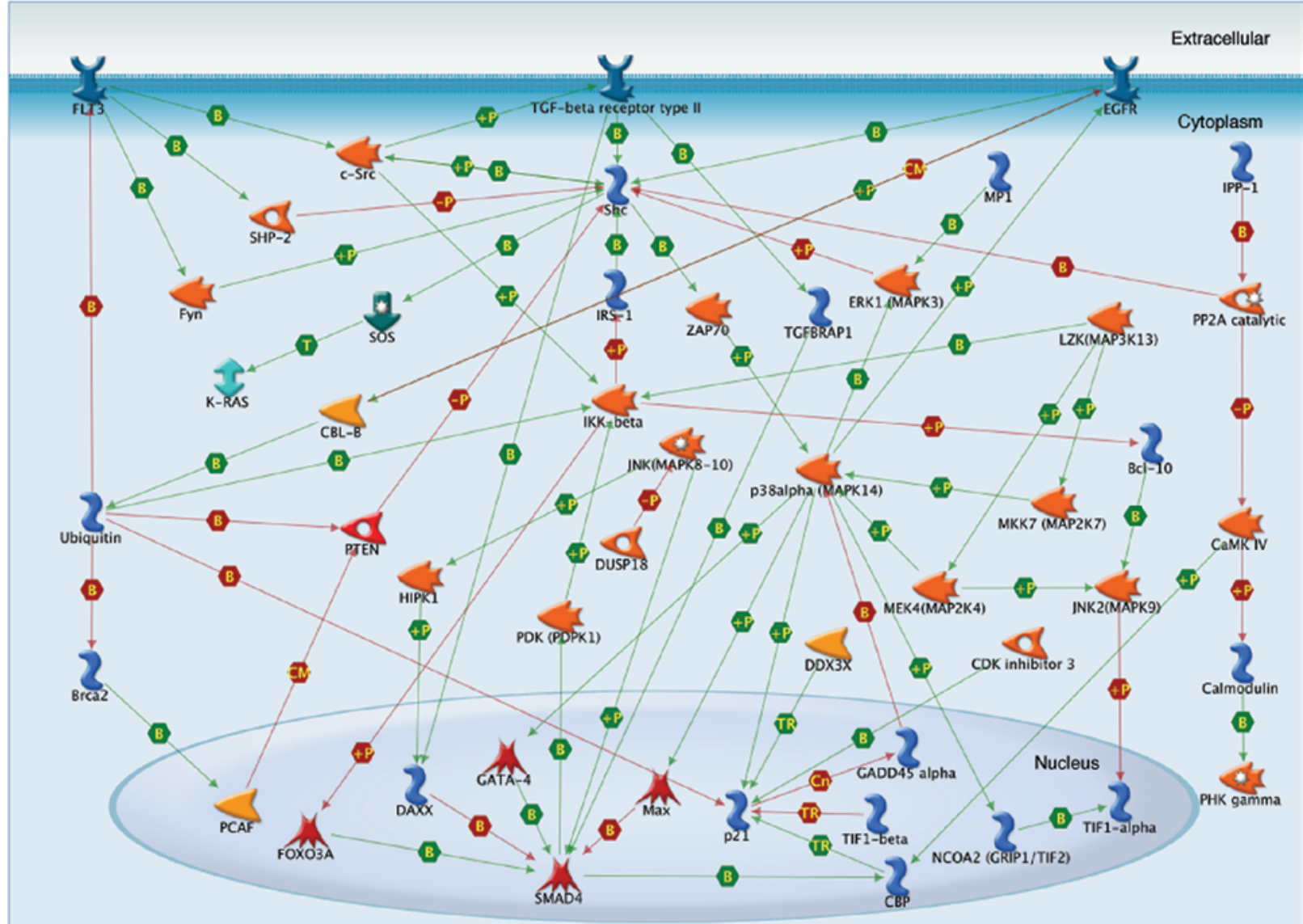

Y

Receptors with enzyme activity

75 RAS-superfamily

B Binding

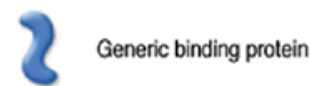

3 Regulators (GDI, GAP, GEF)

CM Covalent modifications

Generic enzyme

Transcription factor

CN compertition

Protein kinase

+ P Phosphorylations

01 Protein phosphatase

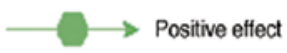

- P Dephosphorylations

Lipid phosphatase

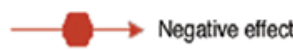

TR

Transcription regulation

B

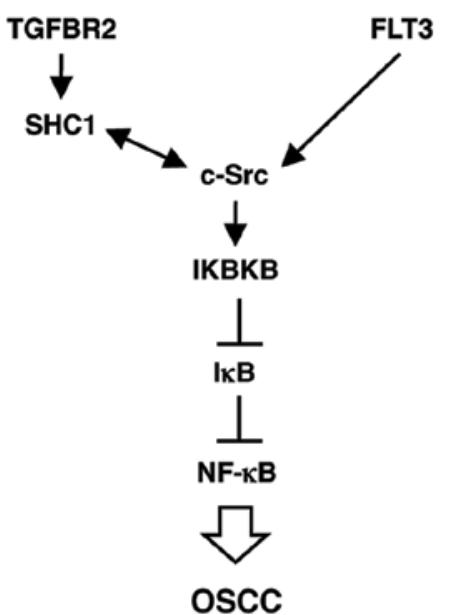

Figure 2. Bioinformatics analysis reveals novel biomarkers and molecular pathways involved in oral cancer HSC-3 cell. (A) Based on the lentivirus-based shRNA high-throughput screening results followed by bioinformatics analysis, candidate biomarkers and their possible molecular pathways were identified. (B) Novel biomarkers and putative molecular siganling pathways involved in the growth regulation of oral cancer HSC-3 cells. 


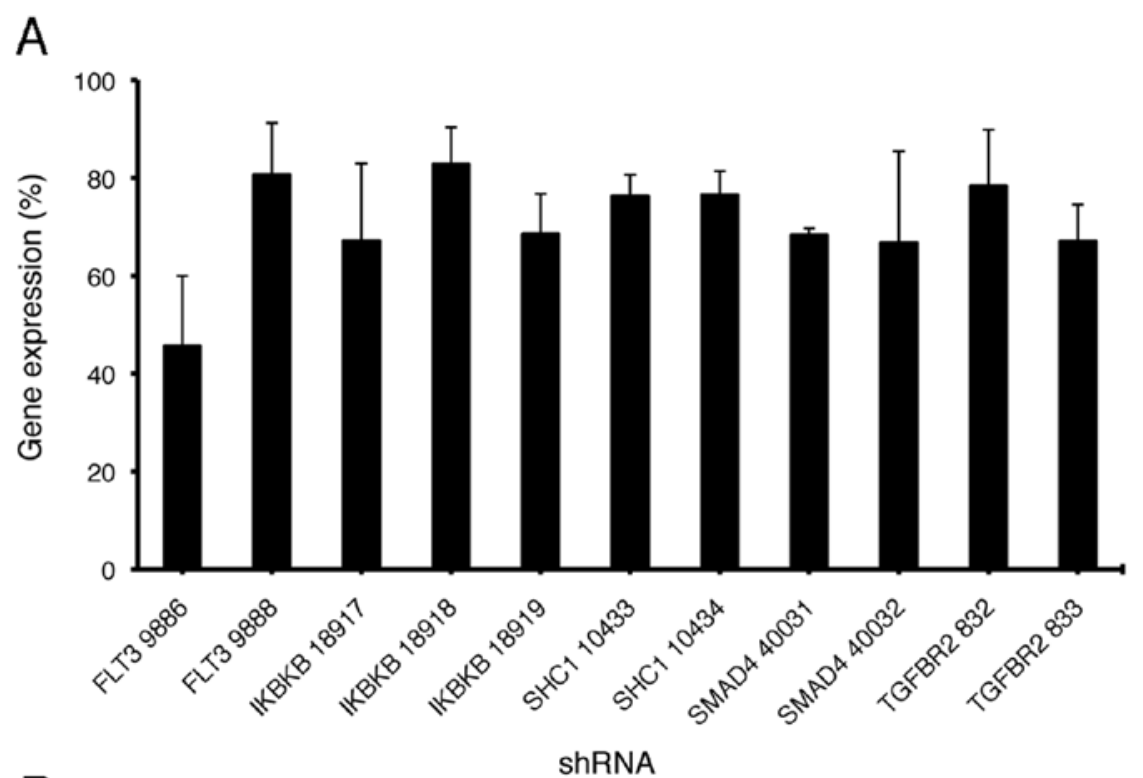

$\mathrm{B}$
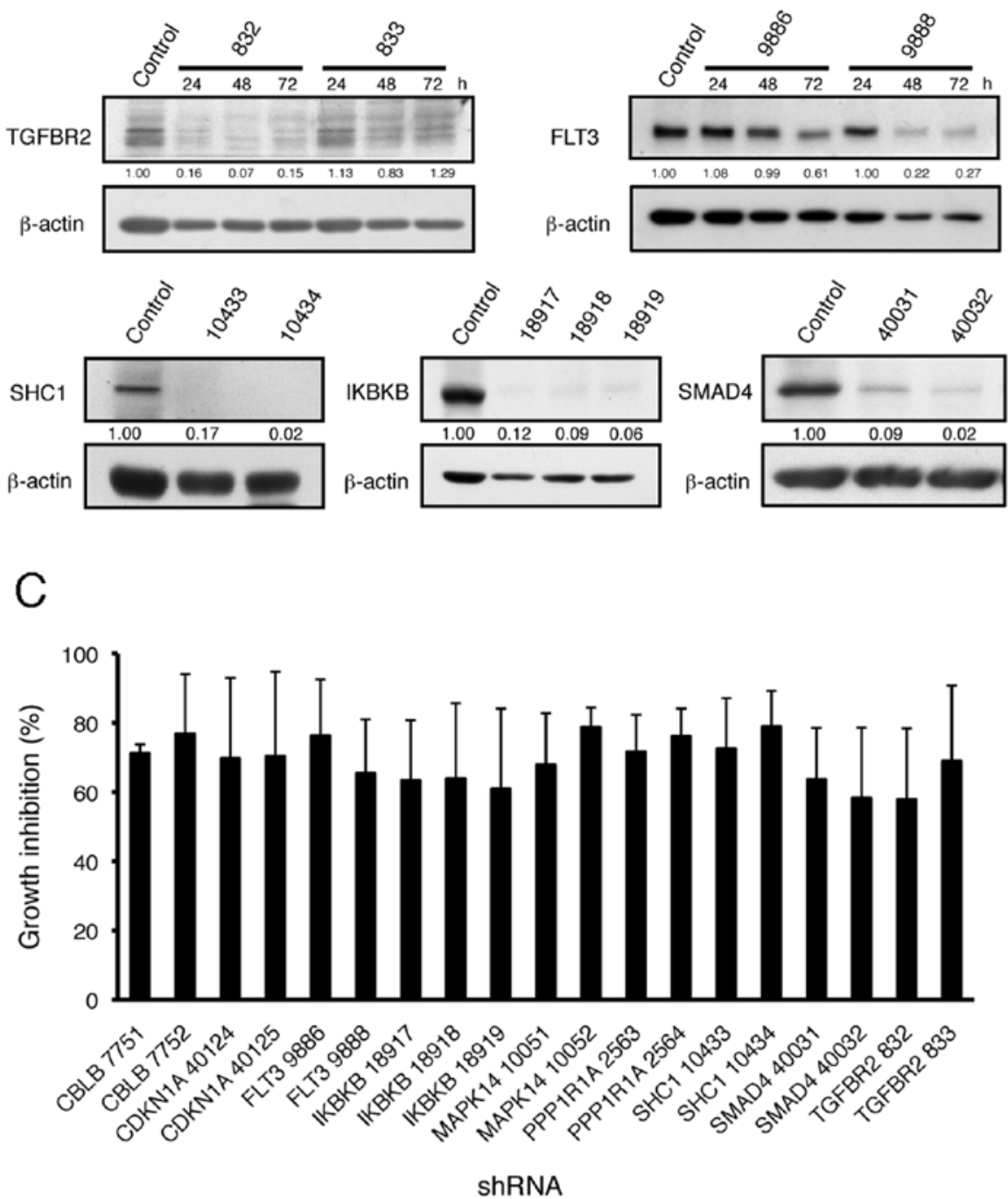

Figure 3. Validation of the candidate shRNAs with taget inhibition effect in HSC-3 cell. Candidate shRNAs were transfected into human oral cancer HSC-3 cells by using liposome as transfection reagent. (A) HSC-3 cells were harvested $48 \mathrm{~h}$ after transfection, followed by total RNA isolation and conversion into cDNA. Subsequently, the gene expression level was measured by real-time PCR and normalized against negative control shLuc. (B) Upper panel, HSC-3 cells were harvested after transfection with TGFBR2 shRNAs (\#832 and \#833) or FLT3 shRNAs (\#9886 and \#9888) for 24, 48 and 72 h. Lower panel, HSC-3 cells were harvested after transfection with shRNAs of SHC1, IKBKB and SMAD4 for $48 \mathrm{~h}$, respectively, and protein expression level was analyzed by Western blotting (C) Growth inhibition of HSC-3 cells relative to control shLuc was measured by CCK- $848 \mathrm{~h}$ after transfection. P $<0.05$, control versus all candidate shRNAs. Data are mean $\pm \mathrm{SD}$ of at least three independent experiments. 

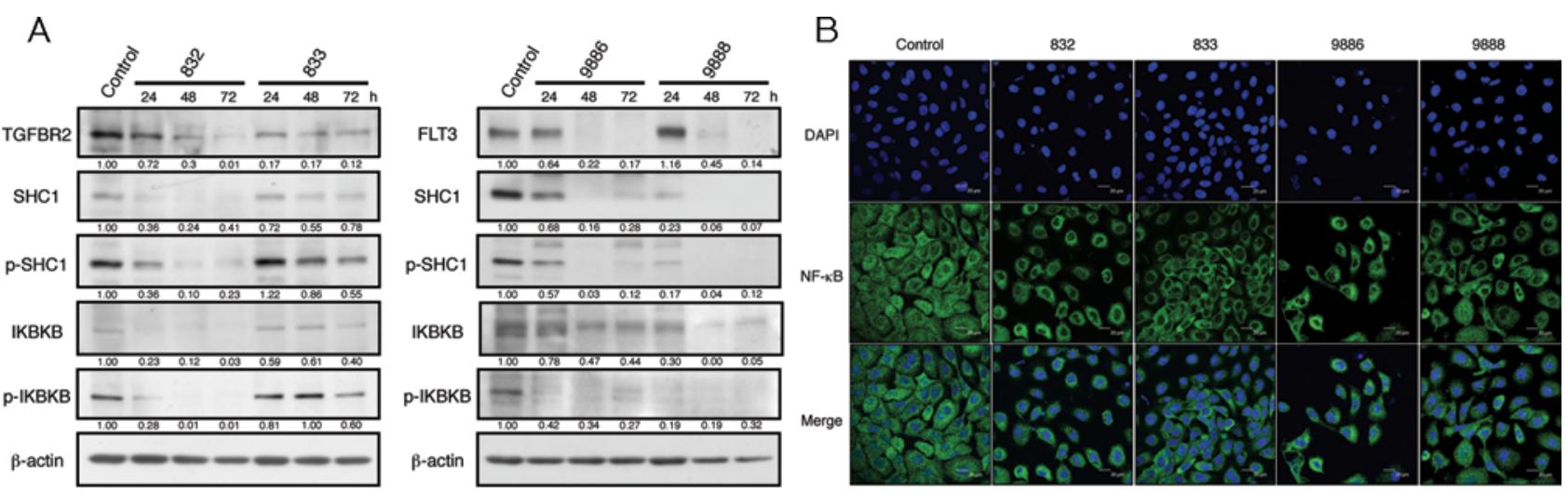

C

LY-364947 ( $\mu \mathrm{M})$

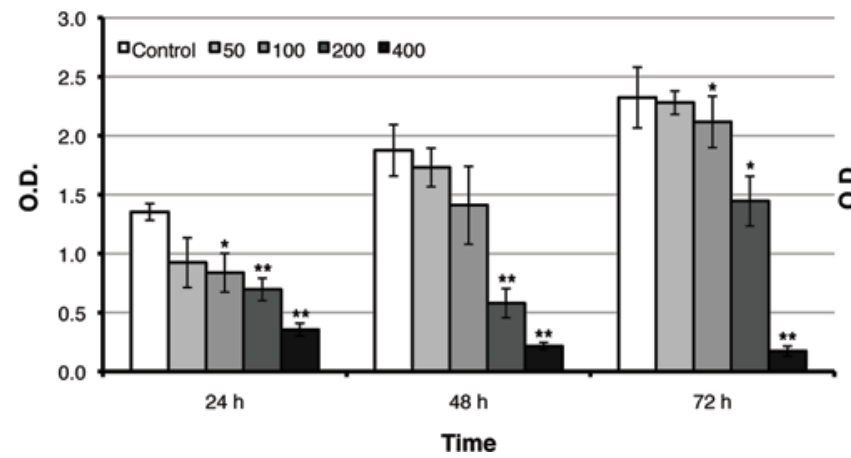

Sunitinib malate $(\mu \mathrm{M})$

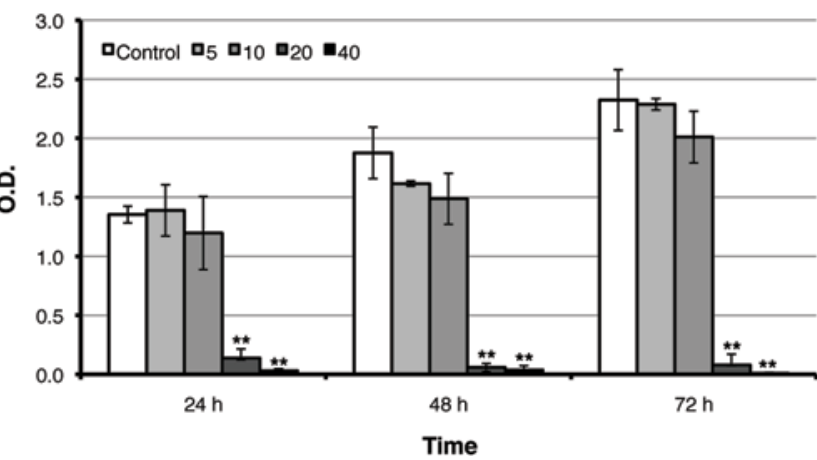

Figure 4. Suppression of TGFBR2 and FLT3 downregulates SHC1 and IKBKB. (A) HSC-3 oral cancer cells were transfected with TGFBR2 shRNAs (\#832 and \#833) or FLT3 shRNAs (\#9886 and \#9888), separately. Protein expression and phosphorylation level of SHC1 and IKBKB were measured by Western blot analysis. (B) HSC-3 cells were fixed after transfection with either TGFBR2 shRNAs (\#832 and \#833) or FLT3 shRNAs (\#9886 and \#9888) and incubation for $48 \mathrm{~h}$, and the subcellular localization of NF-kB was detected by confocal microscopy. (C) HSC-3 cells were treated with tyrosine kinase antagonist LY-364947 $(50,100,200$, and $400 \mu \mathrm{M})$ or Sunitinib malate $(5,10,20$, and $40 \mu \mathrm{M})$ for 24,48 and $72 \mathrm{~h}$, respectively. Cell viability was measured by CCK-8. Results are presented as the mean $\pm \mathrm{SD}$ of at least three independent experiments. ${ }^{*} \mathrm{P}<0.05 .{ }^{* *} \mathrm{P}<0.01$. All assays were performed at least three times.

CBLB, CDKN1A, MAPK14 and SMAD4) were chosen for validation by applying a transient tansfection system to deliver their corresponding shRNAs into HSC-3 cells. First, we examined whether the expression of candidate genes were knocked down by their specific shRNAs. As shown in Fig. 3A and B, all candidate shRNAs were able to suppress both the expression of mRNA and protein of their target genes in HSC-3 cells after $48 \mathrm{~h}$ of transfection. Furthermore, based on the results shown in Fig. 3C, we found these candidate shRNAs could also suppress the growth of HSC-3 cells. These results demonstrated the specificity of candidate shRNAs.

Suppression of TGFBR2 and FLT3 downregulates SHC1 and IKBKB resulting in $N F-\kappa B$ translocalization and growth inhibition of HSC-3 cells. To unravel the putative molecular signaling pathway predicted by bioinformatics analysis, HSC-3 cells were transfected with either TGFBR2 shRNAs (\#832 and \#833) or FLT3 shRNAs (\#9886 and \#9888), respectively, and protein expression and phosphorylation level of SHC1 and IKBKB were measured by Western blot analysis (Fig. 4A) . Both protein expression and phosphorylation level of SHC1 and IKBKB were decreased by TGFBR2 or FLT3 shRNAs. Furthermore, subcellular localization of $\mathrm{NF}-\kappa \mathrm{B}$ was also changed from cytoplasm to nucleus after transfection with either TGFBR2 shRNAs (\#832 and \#833) or FLT3 shRNAs (\#9886 and \#9888) for $48 \mathrm{~h}$ (Fig. 4B). In addition, growth inhibi- tion of HSC-3 cells were also observed by using tyrosine kinase antagonists LY-364947 and Sunitinib malate against TGFBR2 and FLT3, respectively. Taken together, these results revealed a putative molecular signaling pathway which regulates the growth of HSC-3 cells.

\section{Discussion}

With the advancement of genomics technologies, scientists can now easily study various kinds of human disease followed by their molecular signature (29). Among these tools, RNAi provides a comprehensive approach to specifically knock down gene function in a high-throughput fashion in mammalian cells (30). In this study, we used the KP subset to identify potential growth regulators of HSC-3 cells. The screening results identified 50 candidate genes that may be involved in the growth regulation of HSC-3 cells. Inhibition of these candidate genes resulted in significant growth inhibition effect on HSC-3 cells, showing these candidate genes, like oncogenes, may play a role in 'promoting' the growth of OSCC cells. On the other hand, we also identified another group of shRNAs with growth promotion effect on HSC-3 cells (data not shown). These genes, like tumor suppressor genes, may play a role in 'suppressing' the growth of OSCC cells. In order to understand the molecular mechanisms regulating the growth of OSCC cells, both growth promoting and inhibiting groups of genes may be combined for further 
analyzing how they interact each other to regulate the growth of OSCC cells.

The 50 candidate genes were further analyzed by bioinformatics software and revealed a putative molecular signaling pathway regulating the growth of HSC-3 cells. We found that suppression of TGFBR2 or FLT3 expression by specific shRNAs could cause downregulation of protein expression and phosphorylation of SHC1 and IKBKB. Furthermore, suppression of TGFBR2 or FLT3 led to relocalization of transcription factor NF- $\kappa \mathrm{B}$ from the cytoplasm to the nucleus. The human FLT3 gene encodes a membrane-bound receptor tyrosine kinase (RTK), which belongs to the RTK subclass III family. FLT3 is known to play a crucial role in both normal haematopoiesis and acute myeloid leukemia (31). Our results suggest that FLT3 may also be involved in the growth regulation of OSCC cells through regulating IKBKB and $\mathrm{NF}-\kappa \mathrm{B}$. To the best of our knowledge, this is the first report showing that FLT3 plays an important role in the growth regulation of OSCC cells. However, the molecular mechanisms how FLT3 regulates IKBKB and NF- $\kappa$ B remain to be further investigated. Nevertheless, small molecule drugs specifically targeting the RTK subclass III family such as FLT3, like Sunitinib, may hold potential for treating OSCC in the future (32).

The human TGFBR2 gene belongs to the TGF- $\beta$ superfamily receptors. The role of TGF- $\beta$ superfamily members (TGF- $\beta$ s) in carcinogenesis is similar to a double-edged sword. In normal tissues, TGF- $\beta$ s act as tumor suppressors through SMAD-dependent signaling pathways. However, during carcinogenesis, TGF- $\beta$ s perform oncogenic activities, either through SMAD-independent, or cooperation between alternative pathways (e.g., aberrant activation of Ras/MAPK signaling pathways) and relatively low activated SMAD pathways $(33,34)$. Our results show that suppression of TGFBR2 causes growth inhibition of OSCC cells and decreases the protein expression and phosphorylation levels of SHC1 and IKBKB. Furthermore, suppression of TGFBR2 does lead to translocalization of NF- $\kappa B$ without affecting the subcellular localization of SMAD4 (data not shown). Based on these results, it is reasonable to propose that TGFBR2 may promote the growth of OSCC cells through regulating transcription factor $\mathrm{NF}-\kappa \mathrm{B}$.

Among these 50 candidate genes, several genes have been reported to be involved in regulation of apoptosis and chemoresistance in HeLa cervical carcinoma cells (35). These genes are MAP2K1IP1, PPEF2, PTEN and TGFBR2, which were considered as survival genes. Suppression of expression of these genes induced apoptosis or decreased chemoresistance of cancer cells against anticancer drugs like Taxol. However, our results demonstrate that growth inhibition effect caused by suppression of these genes does not induce apoptosis of HSC-3 cells (data not shown). Despite similar effects of these genes on HeLa and HSC-3 cells are not observed; both studies demonstrate that all these genes may still be important biomarkers of cancer cells.

Although PTEN and CDKN1A are known as tumor suppressors, they were also reported to play a role as positive regulators of cell growth (35). Interestingly, in our study we found that suppression of PTEN or CDKN1A resulted in growth inhibition of HSC-3 cells. It is possible that PTEN and CDKN1A may be responsible for other functions in growth regulation of tumor cells. For example, Akt-induced phosphorylation of CDKN1A increases the cytoplasmic localiztion and protein stabillity of CDKN1A, resulting in promotion of growth and survival of tumor cells $(36,37)$. The molecular mechanism of PTEN and CDKN1A in growth regulation of OSCC cells remains to be clarified.

The current strategy to cancer therapy is often referred to as 'one drug for all'. This leads frequently to inappropriate therapy and causes patients to suffer from side effects of drug toxicity. It is believed that personalized medicine has the potential to improve this problem $(18,38)$. In this research, we applied the 'anti-kinome' and 'anti-phosphatome' lentiviral shRNA subset to high-throughput screen and identify the potential biomarkers of OSCC cells. These potential biomarkers and their putative molecular pathways may be used as targets for early diagnosis of the OSCC. Moreover, they may also be beneficial to develop personalized medicine against OSCC.

\section{Acknowledgements}

We acknowledge all lab colleagues and undergraduate students for their efforts and technical assistance on this project. We thank Dr Ru-Chien Cheng (Department of Medical Laboratory Science and Biotechnology, China Medical University, Taichung, Taiwan, R.O.C.) for providing the P2 Lab. We also thank members of the Medical Research Core Facilities Center (Office of Research \& Development, China Medical University, Taichung, Taiwan, R.O.C.) for technical assistance. This work was supported by the China Medical University project CMU95-122 and CMU97090 granted to $\mathrm{MCK}^{*}$, and partly by the Department of Health (DOH-99-TD-C-111-005), Republic of China, granted to NWC.

\section{References}

1. Argiris A, Karamouzis MV, Raben D and Ferris RL: Head and neck cancer. Lancet 371: 1695-1709, 2008.

2 Parkin DM, Bray F, Ferlay J and Pisani P: Global cancer statistics, 2002. CA Cancer J Clin 55: 74-108, 2005.

3. Pfeifer GP, Denissenko MF, Olivier M, Tretyakova N, Hecht SS and Hainaut P: Tobacco smoke carcinogens, DNA damage and p53 mutations in smoking-associated cancers. Oncogene 21: 7435-7451, 2002.

4. Ogden GR: Alcohol and oral cancer. Alcohol 35: 169-173, 2005.

5. Chen YJ, Chang JT, Liao CT, et al: Head and neck cancer in the betel quid chewing area: recent advances in molecular carcinogenesis. Cancer Sci 99: 1507-1514, 2008.

6. Licitra L, Perrone F, Bossi P, et al: High-risk human papillomavirus affects prognosis in patients with surgically treated oropharyngeal squamous cell carcinoma. J Clin Oncol 24: 5630-5636, 2006.

7. Chang NW, Pei RJ, Tseng HC, et al: Co-treating with arecoline and 4-nitroquinoline 1-oxide to establish a mouse model mimicking oral tumorigenesis. Chem Biol Interact 183: 231-237, 2010.

8. Califano J, van der Riet P, Westra W, et al: Genetic progression model for head and neck cancer: implications for field cancerization. Cancer Res 56: 2488-2492, 1996.

9. Campo-Trapero J, Cano-Sanchez J, Palacios-Sanchez B, SanchezGutierrez JJ, Gonzalez-Moles MA and Bascones-Martinez A: Update on molecular pathology in oral cancer and precancer. Anticancer Res 28: 1197-1205, 2008.

10. Kujan O, Oliver RJ, Khattab A, Roberts SA, Thakker N and Sloan P: Evaluation of a new binary system of grading oral epithelial dysplasia for prediction of malignant transformation. Oral Oncol 42: 987-993, 2006.

11. Rosin MP, Cheng X, Poh C, et al: Use of allelic loss to predict malignant risk for low-grade oral epithelial dysplasia. Clin Cancer Res 6: 357-362, 2000.

12. Partridge M, Pateromichelakis S, Phillips E, Emilion GG, A'Hern RP and Langdon JD: A case-control study confirms that microsaellite assay can identify patients at risk of developing oral squamous cell carcinoma within a field of cancerization. Cancer Res 60: 3893-3898, 2000. 
13. Hanahan D and Weinberg RA: The hallmarks of cancer. Cell 100: 57-70, 2000.

14. Blume-Jensen $\mathrm{P}$ and Hunter T: Oncogenic kinase signalling. Nature 411: 355-365, 2001.

15. Ostman A, Hellberg $C$ and Bohmer FD: Protein-tyrosine phosphatases and cancer. Nat Rev Cancer 6: 307-320, 2006.

16. Sheu JJ, Hua CH, Wan L, et al: Functional genomic analysis identified epidermal growth factor receptor activation as the most common genetic event in oral squamous cell carcinoma. Cancer Res 69: 2568-2576, 2009.

17. Hamakawa H, Nakashiro K, Sumida T, et al: Basic evidence of molecular targeted therapy for oral cancer and salivary gland cancer. Head Neck 30: 800-809, 2008.

18. Duffy MJ and Crown J: A personalized approach to cancer treatment: how biomarkers can help. Clin Chem 54: 1770-1779, 2008.

19. Moffat J, Grueneberg DA, Yang X, et al: A lentiviral RNAi library for human and mouse genes applied to an arrayed viral highcontent screen. Cell 124: 1283-1298, 2006.

20. Ekins S, Nikolsky Y, Bugrim A, Kirillov E and Nikolskaya T: Pathway mapping tools for analysis of high content data. Methods Mol Biol 356: 319-350, 2007.

21. Ou CC, Hsu SC, Hsieh YH, et al: Downregulation of HER2 by RIG1 involves the PI3K/Akt pathway in ovarian cancer cells Carcinogenesis 29: 299-306, 2008.

22. Rosnet O, Schiff C, Pebusque MJ, et al: Human FLT3/FLK2 gene: cDNA cloning and expression in hematopoietic cells. Blood 82 1110-1119, 1993.

23. Chuang TC, Liu JY, Lin CT, et al: Human manganese superoxide dismutase suppresses HER 2/neu-mediated breast cancer malignancy. FEBS Lett 581: 4443-4449, 2007.

24. Birmingham A, Selfors LM, Forster T, et al: Statistical methods for analysis of high-throughput RNA interference screens. Nat Methods 6: 569-575, 2009.

25. Dijkstra EW: A note on two problems in connexion with graphs. Numerische Mathematik 1: 269-271, 1959.

26. Neil JR and Schiemann WP: Altered TAB1:I kappaB kinase interaction promotes transforming growth factor beta-mediated nuclear factor-kappaB activation during breast cancer progression. Cancer Res 68: 1462-1470, 2008.
27. Grosjean-Raillard J, Ades L, Boehrer S, et al: Flt3 receptor inhibition reduces constitutive NFkappaB activation in high-risk myelodysplastic syndrome and acute myeloid leukemia. Apoptosis 13: 1148-1161, 2008.

28. Ondrey FG, Dong G, Sunwoo J, et al: Constitutive activation of transcription factors NF-(kappa)B, AP-1, and NF-IL6 in human head and neck squamous cell carcinoma cell lines that express pro-inflammatory and pro-angiogenic cytokines. Mol Carcinog 26: $119-129,1999$.

29. Chen X, Jorgenson E and Cheung ST: New tools for functional genomic analysis. Drug Discov Today 14: 754-760, 2009.

30. Moffat J and Sabatini DM: Building mammalian signalling pathways with RNAi screens. Nat Rev Mol Cell Biol 7: 177-187, 2006.

31. Stirewalt DL and Radich JP: The role of FLT3 in haematopoietic malignancies. Nat Rev Cancer 3: 650-665, 2003.

32. Chow LQ and Eckhardt SG: Sunitinib: from rational design to clinical efficacy. J Clin Oncol 25: 884-896, 2007.

33. Lu SL, Reh D, Li AG, et al: Overexpression of transforming growth factor betal in head and neck epithelia results in inflammation, angiogenesis, and epithelial hyperproliferation. Cancer Res 64: 4405-4410, 2004.

34. Wakefield LM and Roberts AB: TGF-beta signaling: positive and negative effects on tumorigenesis. Curr Opin Genet Dev 12: 22-29, 2002.

35. MacKeigan JP, Murphy LO and Blenis J: Sensitized RNAi screen of human kinases and phosphatases identifies new regulators of apoptosis and chemoresistance. Nat Cell Biol 7: 591-600, 2005.

36. Zhou BP, Liao Y, Xia W, Spohn B, Lee MH and Hung MC: Cytoplasmic localization of $\mathrm{p} 21^{\mathrm{Cip1} / \mathrm{WAF} 1}$ by Akt-induced phosphorylation in HER-2/neu-overexpressing cells. Nat Cell Biol 3 245-252, 2001.

37. Li Y, Dowbenko D and Lasky LA: AKT/PKB phosphorylation of $\mathrm{p} 21^{\mathrm{Cip} / \mathrm{WAF} 1}$ enhances protein stability of $\mathrm{p} 21^{\mathrm{Cip} / \mathrm{WAF} 1}$ and promotes cell survival. J Biol Chem 277: 11352-11361, 2002.

38. Allison M: Is personalized medicine finally arriving? Nat Biotechnol 26: 509-517, 2008. 